\title{
Estimation of glomerular filtration rate by a radial basis function neural network in patients with type-2 diabetes mellitus
}

\author{
Xun Liu ${ }^{1,2+}$, Yan-Ru Chen ${ }^{1 \dagger}$, Ning-shan Li ${ }^{2,3+}$, Cheng Wang ${ }^{1}$, Lin-Sheng $\mathrm{Lv}^{4}$, Ming Li ${ }^{1}$, Xiao-Ming Wu ${ }^{2 *}$
} and Tan-Qi Lou ${ }^{1 *}$

\begin{abstract}
Background: Accurate and precise estimates of glomerular filtration rate (GFR) are essential for clinical assessments, and many methods of estimation are available. We developed a radial basis function (RBF) network and assessed the performance of this method in the estimation of the GFRs of 207 patients with type-2 diabetes and CKD.

Methods: Standard GFR (sGFR) was determined by ${ }^{99 m}$ Tc-DTPA renal dynamic imaging and GFR was also estimated by the 6 -variable MDRD equation and the 4 -variable MDRD equation.

Results: Bland-Altman analysis indicated that estimates from the RBF network were more precise than those from the other two methods for some groups of patients. However, the median difference of RBF network estimates from sGFR was greater than those from the other two estimates, indicating greater bias. For patients with stage I/II CKD, the median absolute difference of the RBF network estimate from sGFR was significantly lower, and the $P_{50}$ of the RBF network estimate $(n=56,87.5 \%)$ was significantly higher than that of the MDRD-4 estimate $(n=49,76.6 \%)$ $(p<0.0167)$, indicating that the RBF network estimate provided greater accuracy for these patients.

Conclusions: In patients with type-2 diabetes mellitus, estimation of GFR by our RBF network provided better precision and accuracy for some groups of patients than the estimation by the traditional MDRD equations. However, the RBF network estimates of GFR tended to have greater bias and higher than those indicated by sGFR determined by ${ }^{99 \mathrm{~m}} \mathrm{Tc}$-DTPA renal dynamic imaging.
\end{abstract}

Keywords: Type 2 diabetes, Chronic kidney disease, Glomerular filtration rate, Artificial neural network

\section{Background}

Diabetic nephropathy is the leading cause of end stage renal disease, a condition characterized by abnormal glomerular filtration rate (GFR) and serum creatinine (SCr) [1]. The National Kidney Foundation (NKF) considers GFR as the best overall measure of kidney function in healthy and diseased individuals [2]. However, measurement of GFR by use of radioisotopes is timeconsuming and expensive, so this method is not used in routine clinical practice. Instead, numerous equations have been proposed to estimate the GFR without the

\footnotetext{
*Correspondence: bmxmwus@scut.edu.cn; lou.tq@163.com

${ }^{\dagger}$ Equal contributors

${ }^{2}$ College of Biology Engineering, South China University of Technology, Guangzhou, China

Full list of author information is available at the end of the article
}

need for radioisotopes [2]. These equations consider $\mathrm{SCr}$ and several additional variables, such as age, gender, race, and body size [2]. The American Diabetes Association [3] also recommends estimation of GFR from serum creatinine (SCr) -based formulae, such as a Modification of Diet in Renal Disease (MDRD) study equation [4].

However, these equations may yield inaccurate estimates in some populations, such as elderly Chinese patients with CKD [5]. Recent studies have criticized the equations currently used to estimate GFR in diabetic patients [6-10]. In particular, the Chronic Kidney Disease Epidemiology Collaboration (CKD-EPI) equation [6], the Mayo Clinic Quadratic (MCQ) equation [7], and the four-variable MDRD equation [8] all underestimated GFR in patients with type- 2 diabetes, and the Cockcroft-

\section{Biomed Central}


Gault equation overestimated GFR in patients with type2 diabetes [10]. These equations may be inaccurate because they do not account for ethnicity [11]. For example, in a group of Chinese patients with CKD, the MDRD equation 7 and the abbreviated MDRD equations underestimated GFR in patients with near-normal renal function and overestimated GFR in patients with advanced renal failure [12]. These equations may also be inaccurate because they were developed by linear regression methods $[11,13,14]$. Linear regression models do not account for the non-linear physiological processes that underlie GFR. Thus, it is important to develop better methodologies for estimation of GFR.

Artificial neural networks (ANNs) have been successfully used to model non-linear phenomena in the field of engineering forecasting. Modern ANNs provide effective nonlinear mapping of data, good fault tolerance, and good self-organization $[15,16]$. Previous research demonstrated that an ANN was more accurate than a logistic regression model in prediction of clinical outcome in patients with systemic inflammatory response syndrome and hemodynamic shock [17]. Other research groups have used ANNs to estimate GFR, including a knowledge-based neural network (KBNN) model [18], an evolving connectionist systems (ECOS) model [19], and a tree-based model with 6 terminal nodes [15]. In all of these cases, the ANNs provided better estimates of GFR than the traditional equations.

Radial basis function (RBF) networks are among the most widely used ANNs, but there have been limited clinical applications of these networks. Our previous study [20] described a simple RBF network for estimation of GFR (eGFR $\mathrm{RBF}_{\text {) }}$ in a group of 327 Chinese patients with chronic kidney disease (CKD). The results indicated that the eGFR $\mathrm{RBF}_{\mathrm{B}}$ had less bias and greater precision than the traditional MDRD equations. The accuracy (deviation less than $30 \%$ from the sGFR) of the $e G_{R B F}$ was significantly better than those from traditional eGFR equations, such as the Jelliffe-1973 -equation and the Ruijin-equation [20].

In the present study, we tested the precision and accuracy of an RBF network model for estimation of GFR in an independent group of 207 Chinese patients who had type- 2 diabetes and CKD and compared the results of the RBF network method with the results from two traditional MDRD formulae [13].

\section{Methods}

\section{Patients}

From January 2005 through December 2009, 207 consecutive patients with type-2 diabetes from the Third Affiliated Hospital of Sun Yat-sen University (Guangzhou, China) were enrolled. Patients younger than 18 years, taking cimetidine or trimethoprim, with acute kidney deterioration, clinical edema, skeletal muscle atrophy, pleural effusion or ascites, malnutrition, amputation, heart failure, or ketoacidosis were excluded. None of the patients were treated by dialysis during the study. CKD was staged according to the National Kidney Foundation (NKF) - Kidney Disease Outcomes Quality Initiative clinical practice guidelines [2] based on the GFR measured by ${ }^{99 m}$ Tc-DTPA dynamic imaging method. Patients were placed into 3 groups based on CKD stage: (i) Stage I/II CKD (GFR $\geq 60 \mathrm{~mL} / \mathrm{min} / 1.73 \mathrm{~m}^{2}$ ); (ii) Stage III CKD $\left(\right.$ GFR $\left.=30-59 \mathrm{~mL} / \mathrm{min} / 1.73 \mathrm{~m}^{2}\right)$; or (iii) Stage IV/V CKD $\left(\right.$ GFR $\left.<30 \mathrm{~mL} / \mathrm{min} / 1.73 \mathrm{~m}^{2}\right)$. The study protocol was approved by the institutional review board at the Third Affiliated Hospital of Sun Yat-sen University and all patients provided informed consent. All participants provided written informed consent.

\section{Measurements}

GFR measured by the ${ }^{99 \mathrm{~m}}$ Tc-DTPA renal dynamic imaging method (modified Gate's method) was used as the standard GFR (sGFR) [21,22], and was calculated as described by $\mathrm{Li}$ et al. [23]. The gamma camera uptake method with ${ }^{99 \mathrm{~m}}$ Tc-DTPA is a simple method for determination of GFR, and is less time-consuming, and less expensive than other methods [24]. Moreover, this method has been recommended as the reference approach for determination of GFR by the Nephrology Committee of Society of Nuclear Medicine [25], and is widely used as a standard method for evaluation of kidney function and estimation of GFR in China. ${ }^{99 \mathrm{~m}} \mathrm{Tc}-$ DTPA renal dynamic imaging was measured by a Millennium TMMPR SPECT using the General Electric Medical System, as described previously [22]. Serum albumin (Alb) and blood urea nitrogen (BUN) were assayed on a Hitachi 7180 autoanalyzer (Hitachi, Tokyo, Japan; reagents from Roche Diagnostics, Mannheim, Germany). SCr was measured by an enzymatic method on the same instrument according to the manufacturer's instructions. SRM967 (standard reference material released by NIST for serum creatinine calibration) was used for calibration. Patient sex, age, height, and weight were recorded at the same time.

\section{RBF network}

An ANN is a computational method composed of interconnected artificial neurons (mathematical functions) that processes information and that typically consists of an input layer, one or more hidden layers, and an output layer. ANNs are used in diverse scientific and engineering fields to model the complex relationships of inputs and outputs. An RBF network is a feed-forward network with one hidden layer, in which activation of the hidden layer is a nonlinear radial basis function 
(a function whose value only depends on the distance to the origin).

In this study, the input layer consisted of measured serum creatinine $(\mathrm{SCr})$ and the output layer consisted of sGFR. Our previous work [20] indicated that when $\mathrm{SCr}$ was measured by the enzymatic method, a simple RBF network model successfully estimated GFR in a population of 327 Chinese patients with CKD, based on analysis of all patients and on analysis of subgroups of patients with different stages of CKD [20]. In this previous study, the RBF network was a feed-forward ANN with an input layer of one unit $(\mathrm{SCr})$, one hidden layer, and an output layer of one unit (sGFR), which was measured in all 327 CKD patients. This RBF network was constructed by use of the newrbe function in MathWorks. In the present study, we tested this RBF network in an independent group of 207 patients who had type- 2 diabetes (external validation data set) to verify the original results.

\section{MDRD equations}

GFR was also estimated by the traditional MDRD equations [13]. In particular, we used the re-expressed 4variable MDRD equation (R-MDRD4):

$$
\begin{aligned}
\mathrm{eGFR}_{4}= & 175 \times \mathrm{Scr}^{-1.154} \times \mathrm{Age}^{-0.203} \\
& \times[0.742 \text { if female }] \times[1.212 \text { if black }]
\end{aligned}
$$

and the re-expressed 6-variable MDRD equation (R-MDRD6):

$$
\begin{aligned}
\mathrm{eGFR}_{6}= & 161.5 \times \mathrm{Scr}^{-0.999} \times \mathrm{Age}^{-0.176} \times \mathrm{BUN}^{-0.17} \\
& \times \mathrm{Alb}^{+0.318} \times[0.762 \text { if female }] \\
& \times[1.18 \text { if black }]
\end{aligned}
$$

\section{Statistical analysis}

All demographic and clinical data are summarized as means \pm standard deviations (SDs), as medians and inter-quartile ranges (IQRs: Q1, Q3) for continuous variables that had non-normal distributions, and as $\mathrm{N}$ and percent for categorical data (CKD stage). Data were compared using a one-way ANOVA with Bonferroni's post hoc correction, the Kruskall Wallis test, the MannWhitney $U$ test for pair-wise comparisons of data that had non-normal distributions, or Pearson's Chi-square test (gender).

The overall differences between eGFR and sGFR are summarized as medians and IQRs due to the nonnormal distributions. Differences among patients with different CKD stages were compared with the Kruskall Wallis test with a post hoc method or with the MannWhitney U test for pair-wise comparisons. Within-group comparisons of measurements were performed using the Wilcoxon signed ranks test for a given CKD stage.
The accuracy of eGFR are summarized as $\mathrm{N}$ and percent of patients with eGFR differing less than $15 \%\left(\mathrm{P}_{15}\right)$, $30 \%\left(\mathrm{P}_{30}\right)$, and $50 \%\left(\mathrm{P}_{50}\right)$ from sGFR. Accuracy of the estimates was compared for patients with different CKD stages using the Pearson Chi-square test. The accuracies of eGFR values were compared using the McNemar test within the same CKD stage. Bland-Altman plots (eGFR vs. sGFR, eGFR ${ }_{6} v s$. sGFR, and $\mathrm{eGFR}_{\mathrm{RBF}} v s$. sGFR) were graphed with Medcalc for Windows (ver. 9.3.9.0, Mariekerke, Belgium). The 3 different methods of estimating GFR were also used to classify patients by CKD stage. A Wilcoxon sign-rank test was used to compare the differences of CKD stages from sGFR and each of these estimates.

All statistical assessments were two-tailed and a $p$-value less than 0.05 was considered significant. The significance level was adjusted by Bonferroni's method to 0.0167 ( 0.05 / 3 ) and $0.01(0.05 / 4)$ for post hoc pair-wise comparisons of CKD stages and eGFR, respectively. All statistical analysis was performed using SPSS (version 11.0 SPSS, Chicago IL, USA).

\section{Results}

A total of 207 patients (119 males, 88 females) with a mean age of 61.43 years $(\mathrm{SD}=12.03)$ were enrolled. Table 1 shows the baseline demographic and clinical characteristics of the 207 patients and of sub-groups with different stages of CKD. There were 64 patients (30.9\%) with stage I/II disease, 81 patients (39.1\%) with stage III disease, and 62 patients (30\%) with stage IV/V disease. As expected, patients with more severe disease had lower serum Alb and sGFR, and higher SCr and BUN (Table 1).

Table 2 shows the sGFR values and the three different estimates of GFR (eGFR 4 , eGFR 6 , and eGFR RBF ) for all 207 patients and for sub-groups with different stages of CKD. As expected, GFR values were lower in patients with more advanced disease. The eGFR $R_{\mathrm{RBF}}$ of all 207 CKD patients and of patients with different stages of CKD were significantly higher than the sGFR values $(p<$ 0.01 for all). In addition, for patients with stage III or

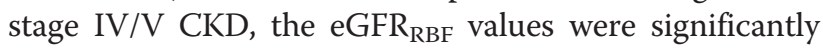
higher than those from eGFR 4 and eGFR $6(p<0.01$ for both).

The validity of an estimate is a function of its accuracy and precision, and a valid estimate should be close to the true value (low bias) and be reproducible [26]. Table 3 presents the overall performances of the 3 different estimates of GFR in all 207 patients and in patients with different stages of CKD. Each row in the table shows the median difference of eGFR and sGFR and the inter-quartile range (IQR), the median absolute difference of eGFR and sGFR and the IQR, and the percent of GFR estimates within $15 \%\left(\mathrm{P}_{15}\right), 30 \%\left(\mathrm{P}_{30}\right)$, and $50 \%$ 
Table 1 Demographic and clinical characteristics of patients with type-2 diabetes and different stages of CKD ( $n=207$ )

\begin{tabular}{|c|c|c|c|c|c|}
\hline Variable & $\operatorname{Total}^{1}(n=207)$ & Stage $I / I I{ }^{1}(n=64)$ & Stage III' $(n=81)$ & Stage IV $/ V^{1}(n=62)$ & P-value \\
\hline Age (years) & $61.43 \pm 12.03$ & $56.64 \pm 11.65$ & $62.78 \pm 11.53^{\mathrm{a}}$ & $64.61 \pm 11.72^{a}$ & $<0.001^{*}$ \\
\hline Sex & & & & & 0.177 \\
\hline Males & $119(57.5)$ & $34(53.1)$ & $53(65.4)$ & 32 (51.6) & \\
\hline Females & $88(42.5)$ & $30(46.9)$ & $28(34.6)$ & $30(48.4)$ & \\
\hline BMI $\left(\mathrm{kg} / \mathrm{m}^{2}\right)$ & $23.42(21.45,25.83)$ & $23.54(21.32,25.77)$ & $22.86(21.16,25.52)$ & $24.05(22.04,25.92)$ & 0.262 \\
\hline $\mathrm{BSA}\left(\mathrm{m}^{2}\right)$ & $1.66(1.54,1.80)$ & $1.66(1.52,1.77)$ & $1.64(1.54,1.81)$ & $1.69(1.54,1.77)$ & 0.826 \\
\hline Alb (g/dL) & $3.75(3.19,4.20)$ & $4.00(3.51,4.41)$ & $3.83(3.30,4.18)$ & $3.39(2.99,3.84)^{\mathrm{ab}}$ & $<0.001^{*}$ \\
\hline $\mathrm{SCr}(\mathrm{mg} / \mathrm{dL})$ & $1.52(0.92,3.72)$ & $0.77(0.57,1.09)$ & $1.58(1.09,2.39)^{\mathrm{a}}$ & $5.19(2.99,7.01)^{\mathrm{ab}}$ & $<0.001^{*}$ \\
\hline BUN (mg/dL) & $27.17(17.76,48.54)$ & $16.70(13.74,21.25)$ & $27.90(18.74,44.82)^{a}$ & $54.68(41.29,70.60)^{\mathrm{ab}}$ & $<0.001^{*}$ \\
\hline DTPA-GFR (mL/min/1.73 $\left.\mathrm{m}^{2}\right)$ & $43.45(25.35,64.36)$ & $78.39(66.86,87.89)$ & $43.42(35.81,54.82)^{a}$ & $19.70(14.58,23.19)^{\mathrm{ab}}$ & $<0.001^{*}$ \\
\hline $\mathrm{GFR} \geqq 60 \mathrm{~mL} / \mathrm{min} / 1.73 \mathrm{~m}^{2}$ & $64(30.9)$ & $64(100)$ & $0(0)$ & $0(0)$ & \\
\hline $\mathrm{GFR}<60 \mathrm{~mL} / \mathrm{min} / 1.73 \mathrm{~m}^{2}$ & $143(69.1)$ & $0(0)$ & $81(100)$ & $62(100)$ & \\
\hline
\end{tabular}

Abbreviations: BMI body mass index, BSA body surface area, Alb albumin, SCr serum creatinine, BUN blood urea nitrogen, DTPA-GFR GFR measured by ${ }^{99 \mathrm{~m}} \mathrm{Tc}$-DTPA renal dynamic imaging.

${ }^{1}$ Continuous data are given as means \pm SDs for age, as medians and IQRs for continuous variables with non-normal distributions, and as $\mathrm{n}$ (\%) for categorical variables. Differences among patients with different stages of CKD were compared with a one-way ANOVA and a Bonferroni post-hoc comparison for age, a Kruskall Wallis test with Mann-Whitney $U$ test for pair-wise comparison of other not normally distributed continuous data; and Pearson's Chi-square test for sex. ${ }^{*} p<0.05$, indicates significant difference among CKD stages.

${ }^{a, b} p<0.0167(0.05 / 3)$, indicates significant difference of CKD stage I/II and III, respectively.

$\left(\mathrm{P}_{50}\right)$ of sGFR [26]. The results indicate that for all 207 patients, the IQR of the eGFR $\mathrm{RBF}_{\text {was }}$ smaller than those from $\mathrm{eGFR}_{4}$ and $\mathrm{eGFR}_{6}$, indicating better precision for the RBF network; however, the median difference of the eGFR $_{\mathrm{RBF}}$ from sGFR was significantly higher than those from eGFR 4 and eGFR $(p<0.0167$ for both). The same trends occurred in patients with stage III CKD and stage

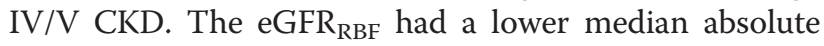
difference from sGFR and a higher percent of estimates within $15 \%\left(\mathrm{P}_{15}\right)$ and $30 \%\left(\mathrm{P}_{30}\right)$ of sGFR, suggesting that eGFR $_{\text {RBF }}$ had better accuracy. However, the differences between eGFR $\mathrm{RBF}_{\text {and }}$ eGFR 4 and eGFR 6 were not statistically different. The results also indicate that for the patients with stage I/II CKD, the median absolute difference of eGFR $\mathrm{RBF}_{\text {and }}$ aGFR (19.73) was significantly less than that of $\mathrm{eGFR}_{4}$ and sGFR $(27.28)(p<0.0167)$, although the $\mathrm{P}_{50}$ for eGFR $\mathrm{RBF}(\mathrm{n}=56,87.5 \%)$ was significantly higher than that for $\mathrm{eGFR}_{4}(\mathrm{n}=49,76.6 \%)(p<$ 0.0167).

Figure 1 shows Bland-Altman plots for comparisons of sGFR with GFR estimated by eGFR 4 (A), eGFR 6 (B), and eGFR $_{\mathrm{RBF}}(\mathrm{C})$. The precision is indicated by the distance between the dashed lines (95\% limits of agreement) [27]. The results indicate that the distance between lines of 95\% limits of agreement was 71.4 for eGFR $_{\mathrm{RBF}}$, significantly lower than that for $\mathrm{eGFR}_{4}$ (91.5) and eGFR 6 (85.2). Thus, this analysis also indicates that the eGFR $\mathrm{RBF}_{6}$ had better precision than the two other estimates from the MDRD equations. However, Figure 1 also shows that the mean differences of sGFR and eGFR 4 was 3.1, sGFR and $\mathrm{eGFR}_{6}$ was 1.0, and sGFR and eGFR $\mathrm{RBF}_{\text {a }}$ was 11.1. This indicates that $\mathrm{eGFR}_{\mathrm{RBF}}$ had greater bias than the estimates from the MDRD equations and over-estimate the GFR.

\section{Discussion}

We compared the performance of an RBF neural network in the estimation of GFR with the performance of two traditional GFR estimates based on the MDRD equations (MDRD-4 and MDRD-6) in patients with type- 2 diabetes and different stages of CKD. Our results indicate that the RBF network provided more precise estimates of GFR than the MDRD equations, and also provided significantly more accurate estimates of GFR for patients with stage I/II CKD. However, the RBF network also had higher bias than the traditional MDRD equa-

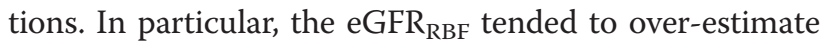
GFR more than $\mathrm{eGFR}_{4}$ and $\mathrm{GFR}_{6}$, especially for patients with CKD stage IV/V (Table 2).

In the field of medical data processing, the theoretical basis for the use of statistical regression methods is the "law of large numbers". That is, the difference of the average of many measurements from the true value should be smaller as more measurements are recorded. However, application of a model derived from one data set to another data set may yield poorer accuracy. Moreover, regression methods can only be used for a limited number of models, and interactions among variables places limits on their use. ANNs have no a priori requirement for data distribution, and can handle multicollinear input variables, neither of which can be managed by regression methods.

These advantages of ANNs have led to their use in several previous studies for estimation of GFR. Song 
Table 2 Glomerular filtration rates (sGFR, eGFR

\begin{tabular}{|c|c|c|c|c|c|}
\hline Measurement method $\left(\mathrm{mL} / \mathrm{min} / 1.73 \mathrm{~m}^{2}\right)$ & Total $^{1}(n=207)$ & Stage $I / / I^{1}(n=64)$ & Stage $\mathrm{III}^{1}(\mathrm{n}=81)$ & Stage IV/V $(n=62)$ & $P$-value \\
\hline SGFR & $43.45(25.35,64.36)$ & $78.39(66.86,87.89)$ & $43.42(35.81,54.82)^{a}$ & $19.70(14.58,23.19)^{\mathrm{ab}}$ & $<0.001^{*}$ \\
\hline $\mathrm{eGFR}_{4}$ & $42.43(15.68,75.46)$ & $89.12(67.73,124.20)^{\dagger}$ & $42.43(26.50,59.31)^{a}$ & $9.60(6.71,17.11)^{\mathrm{ab} t}$ & $<0.001^{*}$ \\
\hline $\mathrm{eGFR}_{6}$ & $39.98(15.28,72.28)^{\ddagger}$ & $90.88(64.52,116.84)^{\ddagger}$ & $40.21(24.72,60.54)^{\mathrm{a} \neq}$ & $9.92(6.54,17.02)^{\mathrm{ab} t}$ & $<0.001^{*}$ \\
\hline eGFR $R_{R B F}$ & $52.25(34.21,81.64)^{\dagger \neq \S}$ & $94.23(70.34,115.27)^{\dagger}$ & $50.42(39.61,70.57)^{\mathrm{a} \ddagger \S}$ & $24.01(18.70,37.28)^{\mathrm{abt} \neq \S}$ & $<0.001^{*}$ \\
\hline
\end{tabular}

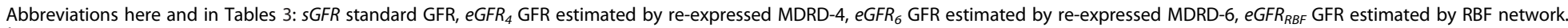

Data are given as medians and IQRs for continuous variables because of non-normal distributions; differences among CKD stages were compared using the Kruskall Wallis test with a post hoc method, or the MannWhitney U test for pair-wise comparisons; within-group comparisons of the four measurements were performed using Wilcoxon Signed Ranks Test for a given CKD stage.

${ }^{*} p<0.05$, indicates significant difference among CKD stages.

$a, b<<0.0167(0.05 / 3)$, indicates significant difference in a comparison of CKD stage I/II and III, respectively.

$t,+, 5, p<0.01(0.05 / 4)$, indicates significant difference in a comparison with sGFR, $\mathrm{GGFR}_{4}$, and eGFR, respectively. 


\section{Table 3 Overall performance of different methods used to estimate glomerular filtration rate in patients with different stages of CKD}

\begin{tabular}{|c|c|c|c|c|c|}
\hline & Median difference of eGFR and sGFR ${ }^{2}$ & Median absolute difference of eGFRand sGFR ${ }^{2}$ & $15 \%\left(P_{15}\right)$ & $30 \%\left(P_{30}\right)$ & $50 \%\left(P_{50}\right)$ \\
\hline \multicolumn{6}{|l|}{ Total $(n=207)$} \\
\hline $\mathrm{eGFR}_{4}$ and $\mathrm{sGFR}{ }^{1}$ & $-2.83(-11.23,12.13)$ & $32.54(15.74,58.97)$ & $49(23.7 \%)$ & $92(44.4 \%)$ & $140(67.6 \%)$ \\
\hline $\mathrm{eGFR}_{6}$ and sGFR ${ }^{1}$ & $-4.06(-12.59,11.21) \dagger$ & $32.78(16.43,57.38)$ & $49(23.7 \%)$ & $91(44.0 \%)$ & $139(67.1 \%)$ \\
\hline eGFR $R_{R B}$ and sGFR & $9.76(-0.19,18.02) \dagger \ddagger$ & $26.24(13.29,55.82)$ & $62(30.0 \%)$ & $112(54.1 \%)$ & $149(72.0 \%)$ \\
\hline \multicolumn{6}{|l|}{ Stage $|/| \mid(n=64)$} \\
\hline $\mathrm{eGFR}_{4}$ and $\mathrm{sGFR}{ }^{1}$ & $9.23(-9.20,37.35)$ & $26.41(12.20,49.65)$ & 19 (29.7\%) & $35(54.7 \%)$ & $49(76.6 \%)$ \\
\hline $\mathrm{eGFR}_{6}$ and $\mathrm{sGFR}{ }^{1}$ & $4.13(-14.66,32.62) \dagger$ & $27.28(12.73,44.42)$ & $16(25.0 \%)$ & $33(51.6 \%)$ & $49(76.6 \%)$ \\
\hline eGFR $R_{R B}$ and sGFR & $12.03(-4.98,27.83)$ & $19.73(12.98,35.56) \dagger$ & $21(32.8 \%)$ & $43(67.2 \%)$ & $56(87.5 \%)+$ \\
\hline \multicolumn{6}{|l|}{ Stage III $(n=81)$} \\
\hline $\mathrm{eGFR}_{4}$ and sGFR 1 & $-1.04(-12.85,9.92)$ & $27.01(13.76,58.47)$ & $22(27.2 \%)$ & $43(53.1 \%)$ & $59(72.8 \%)$ \\
\hline $\mathrm{eGFR}_{6}$ and sGFR ${ }^{1}$ & $-4.80(-14.07,8.97) \dagger$ & $29.82(13.65,57.57)$ & 25 (30.9\%) & $41(50.6 \%)$ & $58(71.6 \%)$ \\
\hline eGFR $R_{R B F}$ and sGFR & $7.96(-1.22,21.47)+\ddagger$ & $25.40(11.23,48.50)$ & $26(32.1 \%)$ & 47 (58.0\%) & $61(75.3 \%)$ \\
\hline \multicolumn{6}{|l|}{ Stage IVN $(n=62)$} \\
\hline $\mathrm{eGFR}_{4}$ and sGFR ${ }^{1}$ & $-6.48(-11.06,-0.21)$ & $46.90(30.81,64.90)$ & $8(12.9 \%)$ & $14(22.6 \%)$ & $32(51.6 \%)$ \\
\hline $\mathrm{eGFR}_{6}$ and $\mathrm{sGFR}{ }^{1}$ & $-6.99(-11.23,-1.63)$ & $46.49(26.56,65.17)$ & $8(12.9 \%)$ & $17(27.4 \%)$ & $32(51.6 \%)$ \\
\hline eGFRRBF and sGFR & $8.89(1.95,15.48) \dagger \neq$ & $49.20(14.98,89.37)$ & 15 (24.2\%) & $22(35.5 \%)$ & $32(51.6 \%)$ \\
\hline
\end{tabular}

Abbreviations as in Table 2 .

${ }^{2}$ Data are given as median difference (IQRs) and median absolute difference (IQRs) between eGFR and three types of eGFR. Within-group comparisons between the measurements were performed using the Wilcoxon Signed Ranks Test for a given CKD stage.

${ }_{*}^{3}$ The accuracy of eGFR was expressed as $n(\%)$ of measurements within 15\% $\left(P_{15}\right), 30 \%\left(P_{30}\right)$, and 50\% $\left(P_{50}\right)$ of sGFR. Data within-measurements were compared using the McNemar test for a given CKD stage.

$p<0.05$, indicates significant difference among CKD stages.

, $\mathrm{P}<0.0167(0.05 / 3)$, indicates significant difference with eGFR ${ }_{4}$ and sGFR and eGFR $\mathrm{R}_{6}$ and sGFR, respectively. 
A

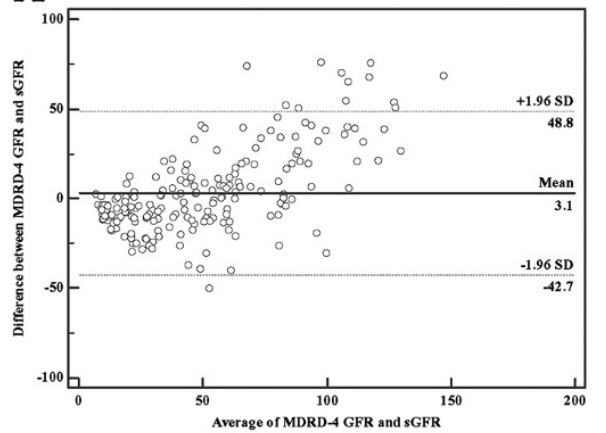

C

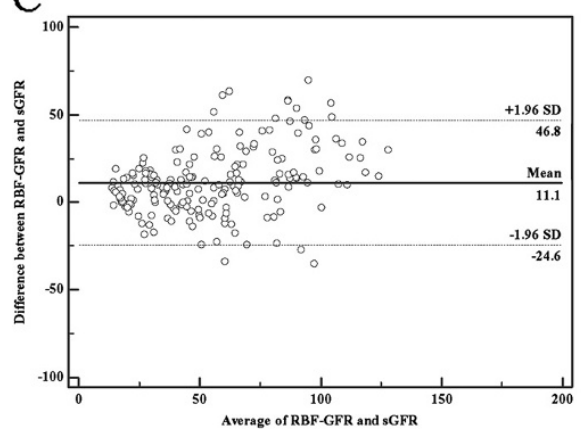

B

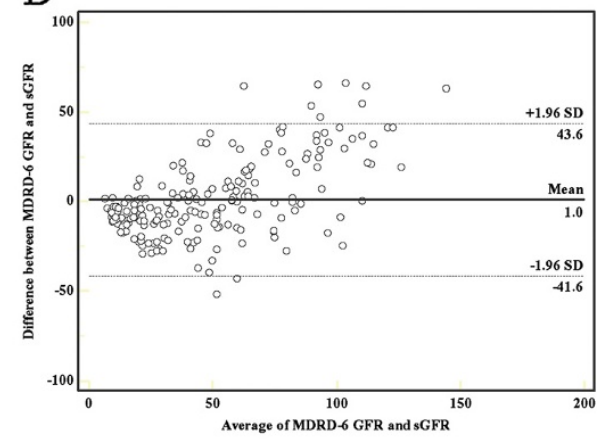

Figure 1 Bland-Altman plots of the estimation of GFR $\left(\mathrm{mL} / \mathrm{min} / 1.73 \mathrm{~m}^{2}\right)$ relative to $\mathrm{SGFR}$ by the (A) re-expressed 4-variable MDRD equation $\left(e G F R_{4}\right)$, (B) re-expressed 6-variable MDRD equation (eGFR $)_{6}$, and (C) RBF network (eGFR RBF ). Solid lines represent mean differences and dashed lines represent $95 \%$ limits of agreement of the mean difference.

et al. [18] used a knowledge-based neural network model (KBNN) for evaluation of renal function based on 441 GFR data vectors from 141 patients. Their proposed GFR prediction model had at least $10 \%$ better accuracy than any of the individual regression formulae or a standard neural network model. Marshall et al. [19] used evolving connectionist systems (ECOS), in which computing structures are trained to generate output from a given set of input variables. They concluded that their ECOS model provided better prediction of GFR in routine clinical practice. No ANNs have been used to estimate GFR of patients with type-2 diabetes mellitus.

It is noteworthy that the RBF network used in this study was only based on SCr measurements, in contrast to the re-expressed MDRD equations, which require measurement of 4 or 6 variables. The NKF does not recommend use of $\mathrm{SCr}$ alone for assessment of kidney function [2]. However, previous research indicated that use of fewer variables can yield acceptable estimates of GFR. For example, Bevc et al. [28] reported that a cystatin C-based estimate, which only requires measurement of serum cystatin $C$, is a reliable marker of GFR in elderly patients and is comparable to the creatininebased formulae, including the CKD-EPI formulae. Our results suggest that an $\mathrm{RBF}$ model based on a single measurement ( $\mathrm{SCr}$ ) can provide precise and accurate estimates of GFR.
There are several limitations to our study. First, SCr was measured by the enzymatic method. Peake et al. [29] indicated that the enzymatic creatinine assay, although theoretically more specific, can have interference problems. However, this method produces results for patient samples that agree closely with the results from isotope dilution mass spectrometry (ID-MS). This motivated our use of the re-expressed MDRD equations (MDRD-4 and MDRD-6) instead of the original equation [4], because the original MDRD equation was developed for use with ID-MS traceable serum creatinine [30]. Second, ANN models can be difficult to display as equations and cannot be readily used without special software, so physicians may be reluctant to accept the results of ANN models. There is need for a platform that can display ANN models and that allows other researchers to readily perform external validation. Third, a previous study-indicated that GFR estimated by $99 \mathrm{mTc}$ DTPA dynamic renal imaging might not better than the modified abbreviated MDRD equation [31], and the renal dynamic imaging method was less accurate than CKD-EPI equation as well [32]. However, the same study found that the two methods performed similar capability in determining GFR among higher-GFR patients [32], and ${ }^{99 \mathrm{~m}}$ Tc-DTPA dynamic renal dynamic imaging yields accurate results that are nearly the same as those from measurements of inulin clearance [33]. Rehling et al. 
showed that a regression line between the values measured by these different methods did not differ from the line of identity [33]. Ma et al. (2007) suggested that, using proper reference GFR, more adequate background subtraction, and soft-tissue attenuation correction may improve the accuracy of ${ }^{99 \mathrm{~m}}$ Tc-DTPA dynamic renal imaging [31]. Finally, our RBF network predicted a higher GFR than that from ${ }^{99 \mathrm{~m}} \mathrm{Tc}$ - DTPA renal dynamic imaging. This might be due to differences of participants in the training group (CKD patients with and without diabetes [20]) and the study group (diabetes patients with and without normal kidney function). Use of more similar training and study groups would provide better external validation and may provide improved results.

A recent survey in China [34] showed that the prevalence of diabetes was $9.7 \%$, corresponding to 92.4 million people. Although some of the established methods used to estimate GFR are suitable for Chinese patients with CKD [22], it is important to have more accurate and precise estimations of GFR. In some measures of accuracy and precision, our RBF neural network performed significantly better than the re-expressed MDRD equations in the estimation of GFR. In particular, the IQRs (Table 3) and 95\% limits of agreement (Figure 1) for the $\mathrm{eGFR}_{\mathrm{RBF}}$ were smaller than those from eGFR 4 and $\mathrm{eGFR}_{6}$, indicating better precision for the RBF network. However, our data indicated that eGFR estimated by the RBF neural network tended to be higher than the sGFR, and this would result in under-estimation of CKD stage. We suggest that use of an RBF network model with more variables and testing of the model with additional data sets may ultimately provide more accurate and precise estimates of GFR.

\section{Conclusions}

In patients with type- 2 diabetes, GFR estimated by our RBF network provided better precision and accuracy for some groups of patients than GFR estimated by the traditional MDRD equations. However, the RBF network estimates of GFR tended to have greater bias and higher than those indicated by sGFR determined by ${ }^{99 \mathrm{~m}} \mathrm{Tc}$ DTPA renal dynamic imaging.

\section{Competing interests}

The author declares that they have no competing interests.

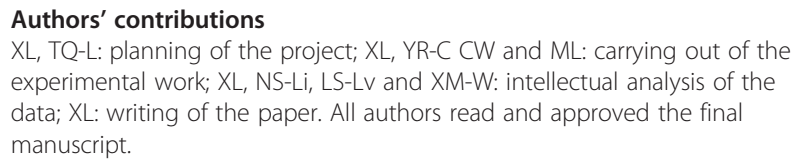

\section{Acknowledgements}

We are grateful to the patients for their cooperation.

\section{Sources of support}

This work were supported by the National Natural Science Foundation of China (Grant No. 81370866 and 81070612), the China Postdoctoral Science
Foundation (Grant No. 201104335), the Guangdong Science and Technology Plan (Grant No. 2011B031800084), the Fundamental Research Funds for the Central Universities (Grant No. 11ykpy38) and the National Project of Scientific and Technical Supporting Programs Funded by Ministry of Science \& Technology of China (Grant No. 2011BAl10B05).

\section{Author details}

${ }^{1}$ Division of Nephrology, Department of Internal Medicine, The Third Affiliated Hospital of Sun Yat-sen University, Guangzhou, China. ${ }^{2}$ College of Biology Engineering, South China University of Technology, Guangzhou, China. ${ }^{3}$ Department of Radiation Oncology, Chengdu International Cancer Treatment Hospital, Chengdu, China. ${ }^{4}$ Operating Room, The Third Affiliated Hospital of Sun Yat-sen University, Guangzhou, China.

Received: 8 October 2012 Accepted: 12 August 2013

Published: 29 August 2013

\section{References}

1. Barnett AH: Preventing renal complications in diabetic patients: the Diabetics Exposed to Telmisartan And enalaprlL (DETAIL) study. Acta Diabetol 2005, 42:S42-S49.

2. National Kidney Foundation: $\mathrm{K} / \mathrm{DOQ}$ clinical practice guidelines for chronic kidney disease: evaluation, classification, and stratification. Am J Kidney Dis 2002, 39:S1-266.

3. American Diabetes Association: Standards of medical care in diabetes2012. Diabetes Care 2012, 35:S11-S63.

4. Levey AS, Bosch JP, Lewis JB, Greene T, Rogers N, Roth D: A more accurate method to estimate glomerular filtration rate from serum creatinine: a new prediction equation. Modification of Diet in Renal Disease Study Group. Ann Intern Med 1999, 130:461-470.

5. Liu X, Wang C, Tang H: Assessing glomerular filtration rate (GFR) in elderly Chinese patients with chronic kidney disease (CKD): A comparison of various predictive equations. Arch Gerontol Geriatr 2010 51:13-20.

6. Silveiro SP, Araújo GN, Ferreira MN, Souza FD, Yamaguchi HM, Camargo EG Chronic Kidney Disease Epidemiology Collaboration (CKD-EPI) equation pronouncedly underestimates glomerular filtration rate in type 2 diabetes. Diabetes Care 2011, 34:2353-2355.

7. Fontseré N, Bonal J, Salinas I, de Arellano MR, Rios J, Torres F, Sanmartí A Romero R: Is the new Mayo Clinic Quadratic equation useful for the estimation of glomerular filtration rate in type 2 diabetic patients? Diabetes Care 2008, 31:2265-2267.

8. Nair S, Mishra V, Hayden K, Lisboa PJ, Pandya B, Vinjamuri S, Hardy KJ, Wilding JP: The four-variable modification of diet in renal disease formula underestimates glomerular filtration rate in obese type 2 diabetic individuals with chronic kidney disease. Diabetologia 2011, 54:1304-1307.

9. Joshy G, Porter T, Le Lievre C, Lane J, Williams M, Lawrenson R: Implication of using estimated glomerular filtration rate (GFR) in a multi ethnic population of diabetes patients in general practice. N Z Med J 2010, 123:9-18.

10. Rigalleau V, Beauvieux MC, Le Moigne F, Lasseur C, Chauveau P, Raffaitin C, Perlemoine C, Barthe N, Combe C, Gin H: Cystatin C improves the diagnosis and stratification of chronic kidney disease, and the estimation of glomerular filtration rate in diabetes. Diabetes Metab 2008, 34:482-489.

11. Stevens LA, Claybon MA, Schmid CH, Chen J, Horio M, Imai E, Nelson RG, Van Deventer M, Wang HY, Zuo L, Zhang YL, Levey AS: Evaluation of the Chronic Kidney Disease Epidemiology Collaboration equation for estimating the glomerular filtration rate in multiple ethnicities. Kidney Int 2011, 79:555-562.

12. Zuo L, Ma YC, Zhou YH, Wang M, Xu GB, Wang HY: Application of GFRestimating equations in Chinese patients with chronic kidney disease. Am J Kidney Dis 2005, 45:463-472.

13. Levey AS, Coresh J, Greene T, Stevens LA, Zhang YL, Hendriksen S, Kusek $J W$, Van Lente F: Using standardized serum creatinine values in the modification of diet in renal disease study equation for estimating glomerular filtration rate. Ann Intern Med 2006, 145:247-254.

14. Levey AS, Stevens LA, Schmid CH: CKD-EPI (Chronic Kidney Disease Epidemiology Collaboration). A New Equation to Estimate Glomerular Filtration Rate. Ann Intern Med 2009, 150:604-612. 
15. Goldfarb-Rumyantzev AS, Pappas L: Prediction of renal insufficiency in Pima Indians with nephropathy of type 2 diabetes mellitus. Am J Kidney Dis 2002, 40:252-264.

16. Baxt WG, Skora J: Prospective validation of artificial neural network trained to identify acute myocardial infarction. Lancet 1996, 347:12-15

17. Dybowski R, Weller P, Chang R, Gant V: Prediction of outcome in critically ill patients using artificial neural network synthesised by genetic algorithm. Lancet 1996, 347:1146-1150.

18. Song Q, Kasabov N, Ma T, Marshall MR: Integrating regression formulas and kernel functions into locally adaptive knowledge-based neural networks: a case study on renal function evaluation. Artif Intell Med 2006, 36:235-244.

19. Marshall MR, Song Q, Ma TM, MacDonell SG, Kasabov NK: Evolving connectionist system versus algebraic formulas for prediction of renal function from serum creatinine. Kidney Int 2005, 67:1944-1954.

20. Liu X, Wu XM, Li LS, Lou TQ: Application of radial basis function neural network to estimate glomerular filtration rate in Chinese patients with chronic kidney disease. ICCASM 2010, 15:332-335 [The International Conference on Computer Application and System Modeling].

21. Du X, Liu L, Hu B, Wang F, Wan X, Jiang L, Zhang R, Cao C: Is the Chronic Kidney Disease Epidemiology Collaboration four-level race equation better than the cystatin C equation? Nephrology (Carlton) 2012, 17:407-414.

22. Liu X, Lv L, Wang C, Shi C, Cheng C, Tang H, Chen Z, Ye Z, Lou T: Comparison of prediction equations to estimate glomerular filtration rate in Chinese patients with chronic kidney disease. Intern Med J 2012, 42:e59-67.

23. Li Q, Zhang CL, Fu ZL, Wang RF, Ma YC, Zuo L: Development of formulae for accurate measurement of the glomerular filtration rate by renal dynamic imaging. Nucl Med Commun 2007, 28:407-413.

24. Assadi M, Eftekhari M, Hozhabrosadati M, Saghari M, Ebrahimi A, Nabipour I, Abbasi MZ, Moshtaghi D, Abbaszadeh M, Assadi S: Comparison of methods for determination of glomerular filtration rate: low and high-dose Tc99m-DTPA renography, predicted creatinine clearance method, and plasma sample method. Int Urol Nephrol 2008, 40:1059-1065.

25. Blaufox MD, Aurell M, Bubeck B, Fommei E, Piepsz A, Russell C, Taylor A, Thomsen HS, Volterrani D: Report of the Radionuclides in Nephrourology Committee on renal clearance. J Nucl Med 1996, 37:1883-1890.

26. Stevens $L A$, Zhang $Y$, Schmid $\mathrm{CH}$ : Evaluating the performance of equations for estimating glomerular filtration rate. J Nephrol 2008 21:797-807.

27. Bland JM, Altman DG: Statistical methods for assessing agreement between two methods of clinical measurement. Lancet 1986, 1:307-310.

28. Bevc S, Hojs R, Ekart R, Gorenjak M, Puklavec L: Simple cystatin C formula compared to sophisticated CKD-EPI formulas for estimation of glomerular filtration rate in the elderly. Ther Apher Dial 2011, 15:261-268.

29. Peake $M$, Whiting M: Measurement of serum creatinine- current status and future goals. Clin Biochem Rev 2006, 27:173-184.

30. Hallan S, Astor B, Lydersen S: Estimating glomerular filtration rate in the general population: the second Health Survey of Nord-Trondelag (HUNT II). Nephrol Dial Transplant 2006, 21:1525-1533.

31. Ma YC, Zuo L, Zhang CL, Wang M, Wang RF, Wang HY: Comparison of 99mTc-DTPA renal dynamic imaging with modified MDRD equation for glomerular filtration rate estimation in Chinese patients in different stages of chronic kidney disease. Nephrol Dial Transplant 2007, 22:417-23.

32. Xie P, Huang JM, Liu XM, Wu WJ, Pan LP, Lin HY: (99m)Tc-DTPA renal dynamic imaging method may be unsuitable to be used as the reference method in investigating the validity of CKD-EPI equation for determining glomerular filtration rate. PLoS One 2013, 8:e62328.
33. Rehling M, Møller ML, Thamdrup B, Lund JO, Trap-Jensen J: Reliability of a 99mTc-DTPA gamma camera technique for determination of single kidney glomerular filtration rate. A comparison to plasma clearance of $51 \mathrm{Cr}$-EDTA in one-kidney patients, using the renal clearance of inulin as a reference. Scand J Urol Nephrol 1986, 20:57-62.

34. Yang W, Lu J, Weng J, Jia W, Ji L, Xiao J, Shan Z, Liu J, Tian H, Ji Q, Zhu D, Ge J, Lin L, Chen L, Guo X, Zhao Z, Li Q, Zhou Z, Shan G, He J, China National Diabetes and Metabolic Disorders Study Group: Prevalence of diabetes among men and women in China. N Engl J Med 2010, 362:1090-1101.

doi:10.1186/1471-2369-14-181

Cite this article as: Liu et al:: Estimation of glomerular filtration rate by a radial basis function neural network in patients with type- 2 diabetes mellitus. BMC Nephrology 2013 14:181.

\section{Submit your next manuscript to BioMed Central and take full advantage of:}

- Convenient online submission

- Thorough peer review

- No space constraints or color figure charges

- Immediate publication on acceptance

- Inclusion in PubMed, CAS, Scopus and Google Scholar

- Research which is freely available for redistribution
C Biomed Central 